\title{
Synthesis, Structural and Biochemical Aspects of Zinc(II) Complexes of Oxadiazole Functionalised Dithiocarbazinates
}

\author{
S.K. Sengupta", O.P. Pandey and A.K. Singh \\ Department of Chemistry, D.D.U. Gorakhpur University, Gorakhpur-273009, India \\ E-mail: sengupta2002(äyahoo.co.in
}

\begin{abstract}
A novel series of complexes, of the type $\left[\mathrm{Zn}(\mathrm{L})_{2}\right]$ ( $\mathrm{LK}=$ potassium salt of $\mathrm{N}$-(5-phenyl/ o-chlorophenyl/pchlorophenyl/o-methylphenyl/o-nitrophenyl-1,3,4-oxadiazole-2-yl)dithiocarbazinate) have been synthesized and characterized by elemental analyses, conductance measurements and spectral (u.v., IR and 'H NMR) data. The ligands behave as bidentate chelating agents coordinating through two sulphur atoms. Probable structures are proposed for the complexes. Attempts have been made to establish a correlation between biological activity (antifungal and antibacterial) and the structures of the products.
\end{abstract}

\section{INTRODUCTION}

In recent years, zinc complexes with sulphur donor ligands have been the centre of attention since zincsulphur coordinated bonds have a wide range of applications $/ 1 /$. Our interest in the synthesis and characterization of dithiocarbazinate ligands is due to their similarities with the dithiocarbamate complexes. Dithiocarbamato complexes have been reported for a great number of metal ions, and they have been used extensively in analytical chemistry $/ 2-4 /$. They also have been used with success as fungicides, pesticides, vulcanisation accelerators, flotation agents and high pressure lubricants $15-10 \%$. In all these derivatives, the dithiocarbamato group coordinates through two sulphur atoms resulting in the formation of four-membered chelate rings. It has also been observed that the dithiocarbamato ligands, by virtue of their low charge and small bites $(\sim 2.8-2.9 \AA)$, are well suited for stabilization of higher coordination states of metals. Coordination behaviour of dithiocarbamate ligands containing heterocyclic ring at nitrogen has also been reported /11,12/. In such cases, the ligands coordinate through nitrogen and sulphur, rather than both sulphur atoms, as observed in simple dithiocarbamate ligands. However, no paper has appeared on coordination behaviour of analogous dithiocarbazinate ligands $\left(\mathrm{X}=-\mathbf{N H}-\mathrm{NR}_{2}\right)$. These ligands contain an additional $\mathbf{N H}$ group between $\mathrm{N}$ and $\mathrm{C}$. On the other hand, 1,3,4-oxadiazole ring exhibits relevant biological properties, which have given rise to a wide variety of applications, in particular as active compound in both medicine and agriculture $/ 13,14 /$. 
The present paper includes the reactions of zinc acetate with one new series of dithiocarbazinate ligands (I), in which one heterocyclic ring (oxadiazole) is attached to nitrogen.<smiles>[R][X]1ccc(-c2nnc(NNC(=S)[Hg])o2)cc1</smiles>

(I)

$\mathrm{R}=\mathrm{H}$ (PODC.K); 2-Cl (OCODC.K); 4-Cl (PCODC.K); 2-CH 3 (MODC.K); 4-NO 2 (NODC.K)

\section{EXPERIMENTAL}

The solvents were purchased from Merck and used without further purification. Zinc acetate dihydrate was purchased from Aldrich. The ligands were prepared as reported in the literature ${ }^{15}$. Elemental analyses and physical measurements were made as noted earlier ${ }^{16}$.

\section{Preparation of complexes}

A general procedure was followed to synthesize these complexes. The procedure involves the addition of the appropriate ligand $(0.04 \mathrm{~mol})$ to an aqueous ethanolic solution of zinc acetate dihydrate $(0.02 \mathrm{~mol})$. The mixture was refluxed for $c a .6-7 \mathrm{~h}$ on a water bath. Light yellow or brown precipitate obtained was filtered, washed with ethanol and hot water and dried in vacuo at room temperature.

\section{RESULTS AND DISCUSSION}

The reactions of zinc(II) acetate dehydrate with appropriate dithiocarbazinate (LK), such as PODC.K = potassium N-(5-phenyl-1,3,4-oxadiazole-2-yl)dithiocarbazinate, OCODC.K $=$ potassium N-(5-ochlorophenyl-1,3,4-oxadiazole-2-yl)dithiocarbazinate, PCODC.K = potassium N-(5-p-chlorophenyl-1,3,4oxadiazole-2-yl)dithiocarbazinate, MODC.K = potassium N-(5-o-methylphenyl-1,3,4-oxadiazole-2yl)dithiocarbazinate ,NODC.K = potassium N-(5-p-nitrophenyl-1,3,4-oxadiazole-2-yl)- dithiocarbazinate have been studied on refluxing in ethanol in molar ratio 1:2, respectively. The compounds of the types $\left[\mathrm{Zn}(\mathrm{L})_{2}\right]$ were obtained. The reactions can be represented by the following equations:

$$
\begin{aligned}
& \mathrm{Zn}\left(\mathrm{CH}_{3} \mathrm{COO}\right)_{2}+2 \mathrm{L.K} \underset{\text { reflux }}{\stackrel{\mathrm{EtOH}}{\longrightarrow}}\left[\mathrm{Zn}(\mathrm{L})_{2}\right]+2 \mathrm{CH}_{3} \mathrm{COOK} \\
& \text { (LK= PODC.K, OCODC.K, PCODC.K, MODC.K, NODC.K) }
\end{aligned}
$$

The physical properties and the analytical data of the complexes are given in Table I. 


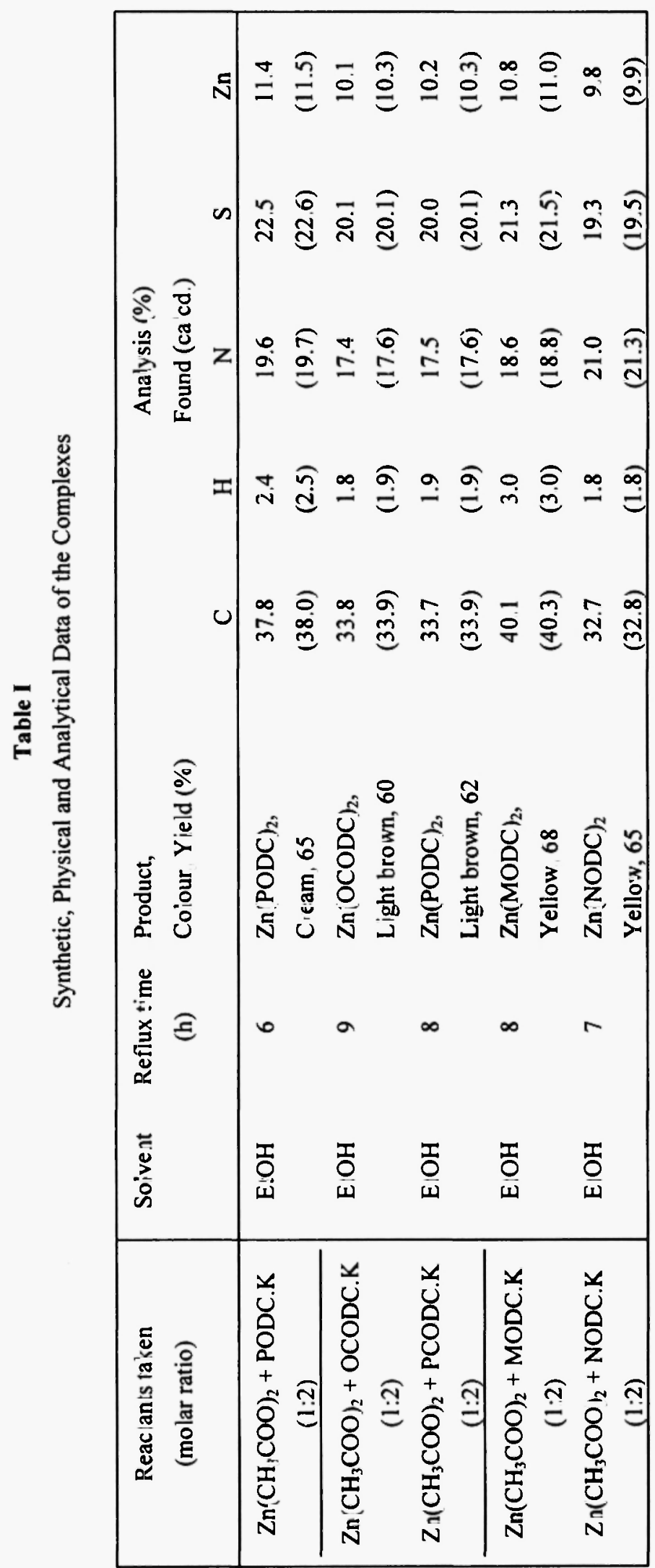


The methods used for the preparation and isolation of these compounds give materials of good purity, as supported by their analysis and thin layer chromatography. These complexes are light yellow or brown coloured solids. They are quite stable in air and decompose in the temperature range $167-250^{\circ} \mathrm{C}$. Complexes are soluble in tetrahydrofuran, dimethylformamide, dimethylsulphoxide and nitrobenzene. The electrical conductance measurements in nitrobenzene indicate that complexes are non-electrolytic in nature. Magnetic susceptibility values at room temperature show their diamagnetic nature.

\section{ELECTRONIC SPECTRA}

The electronic spectra of the ligands and complexes show two bands at $c a .32,000$ and $34500 \mathrm{~cm}^{-1}$, due to intra-ligand transitions.

\section{INFRARED SPECTRA}

The significant infrared frequencies of complexes are given in Table II. The major interest in preparation of these complexes is to study the effect of the attachment of dithiocarbazinate ligand to zinc metal in presence of the bulky oxadiazole ring, which due to some steric factors, might have some effects on the structure of the complexes. A number of workers carried out extensive studies $/ 17-20 /$ on the mode of bonding of dithiocarbamate ligands to the metal. The different resonating structures (II) of the dithiocarbamate moiety can be represented as below:

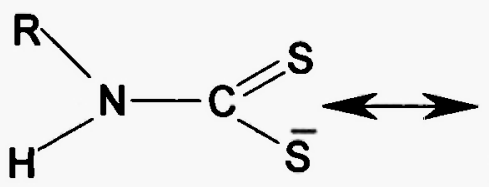

(a)

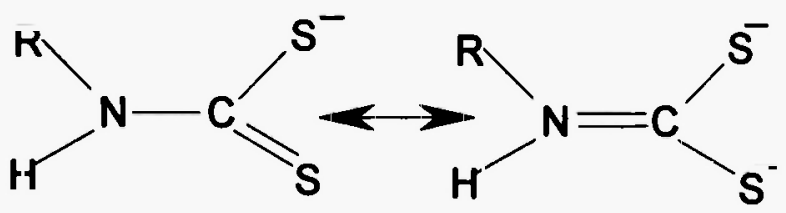

(b) (c)

(II)

The extent to which these resonance forms contribute to the structure, and its effect on the physical and chemical properties, has been extensively studied. Chatt et al. on the basis of detailed infrared spectral studies of a large number of dithiocarbamate complexes, suggested /21/ that the resonance form (c) does indeed contribute to the structure to a considerable extent, but resonance forms (a) and (b) too contribute equally to the structure, provided dipole moment studies are also taken into consideration. Finally, Nakamoto et al., carried out the normal coordinate analysis of the dithiocarbamato metal complexes and concludeD /22/ that the contribution of the resonance form (c) is greater than the others to the total structure of dithiocarbamato complexes and it increases appreciably in $\mathrm{N}$-alkyl derivatives. 


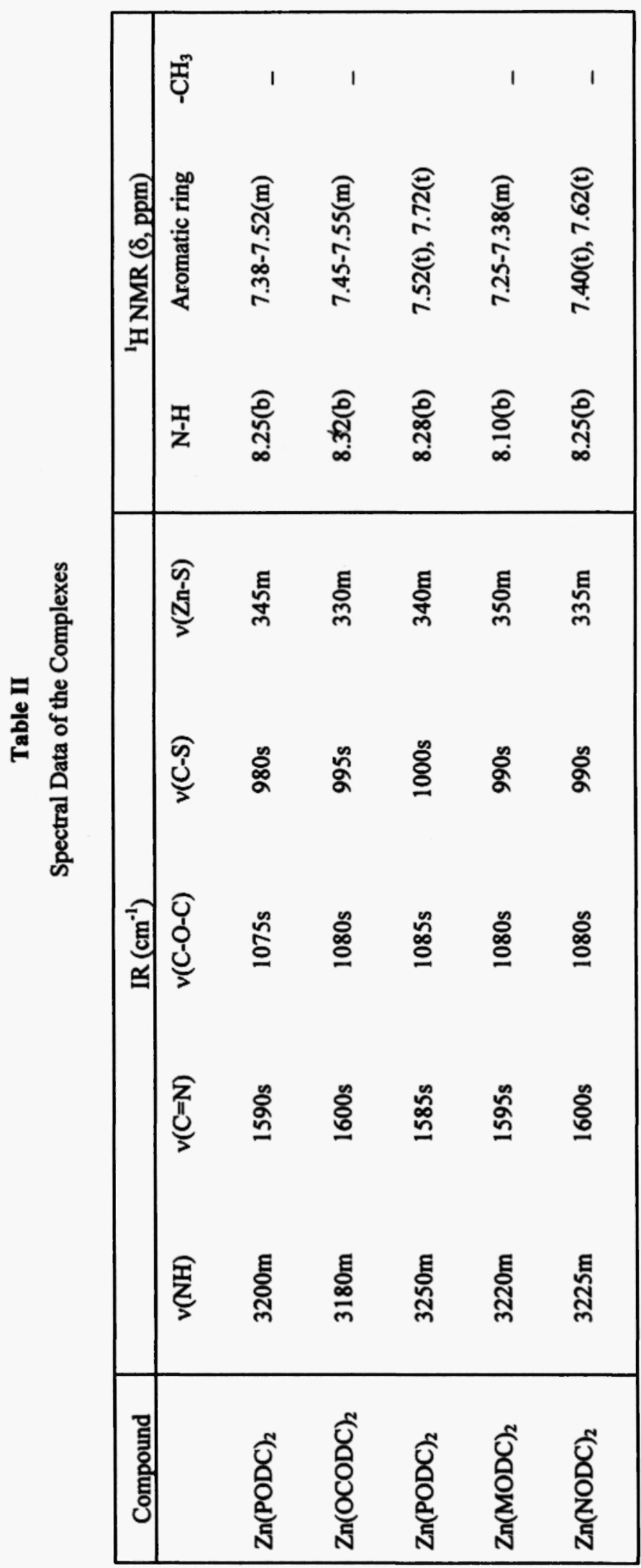


The strong band appearing near $1500 \mathrm{~cm}^{-1}$ in the infrared spectra of the ligands can be assigned to the "thioureid" group, $\mathrm{S}=\mathrm{C}-\mathrm{N}^{-}$. The energy of this band lies intermediate to the stretching frequencIES associated to $\mathrm{C}-\mathrm{N}$ bond $\left(1250-1350 \mathrm{~cm}^{-1}\right)$ and to $\mathrm{C}=\mathrm{N}$ bond $\left(1640-1690 \mathrm{~cm}^{-1}\right)$, and can best to explained as a vibration of a polar $\mathrm{C}=\mathrm{N}^{+}$bond. Thus, the "thioureid" band near $1500 \mathrm{~cm}^{-1}$ implies a considerable double bond character in the $\mathrm{S}_{2} \mathrm{C}-\mathrm{NRR}^{\prime}$ bond. In general, $\mathrm{C}-\mathrm{N}$ frequency shows a blue shift in the complexes as compared to the respective dithiocarbamate ligand, if the dithiocarbamate group behaves as a bidentate ligand. For a monodentate dithiocarbamate moiety, this frequency should exhibit either no change in position or undergo red shift with respect to the corresponding free ligand frequencY $/ 11,12 /$. In the dithiocarbazinate ligands, this band appears at $c a .1510 \mathrm{~cm}^{-1}$. In the complexes cited here, this band undergoes blue shift indicating the bidentate nature of the ligand. The monodentate or bidentate nature of dithiocarbamato group in the ligand is further reflected in the (C-S) stretching frequency. In the case of bidentate behaviour, a single strong band appears in the region of $970-1000 \mathrm{~cm}^{-1}$, while a doublet is expected in the region of $1000 \mathrm{~cm}^{-1}$ for the monodentate one. The complexes reported show a single band at $\mathrm{ca} .990$ $\mathrm{cm}^{-1}$, thus indicating the coordination of both sulphurs to metal. The $\mathrm{v}(\mathrm{Zn}-\mathrm{S})$ vibrations appear at $c a .330-350$ $\mathrm{cm}^{-1}$.

A strong band in the region of $1585-1600 \mathrm{~cm}^{-1}$ in the ligands is characteristic $/ 11 /$ of the $v(C=N)$ group. In the complexes the $\mathrm{v}(\mathrm{C}=\mathrm{N})$ band remains at the same position, indicating the non-coordination of ring nitrogen to metal. The $v(\mathrm{NH})$ band appears at $-3180-3250 \mathrm{~cm}^{-1}$ in the ligands. The persistence of this band at the same position in the complexes indicates the non-coordination of the amino nitrogen.

The v(C-O-C) vibration appears as a strong band at $c a .1080 \mathrm{~cm}^{-1}$ in the free ligands; the position of which also remains the same in the complexes, indicating $/ 23,24 /$ non-coordination of oxadiazole ring oxygen to metal atom.

Thus, the infrared spectra suggest that the both the sulphur atoms of the dithiocarbazinate moiety are involved in chelation.

\section{PROTON MAGNETIC RESONANCE SPECTRA}

The proton magnetic resonance spectra of these complexes have been recorded in deuterated dimethylsulphoxide. Chemical shifts for protons in different environments have been given in Table II. The intensities of all the resonance lines were determined by planimetric integration. The following conclusions can be derived by comparing the spectra of ligands with their corresponding complexes:

(i) In general, a slight lower field shift in the positions of the resonance signals of various protons ( $R$, $\mathrm{NH}$ ) in the complexes were observed due to a change in the electronic environments (deshielding) around the $\mathrm{R}, \mathrm{NH}$ protons in the ligands on coordination with the metal atom. Of course, the protons of these groups in the ligands are very little affected, due to the remote positions of these protons from the coordinating atoms in these complexes.

(ii) A broad NH peak appears in all the complexes near $\delta 8.15-8.35$.

(iii) For phenyl ring, a multiplet appears at ca. $\delta$ 7.25-7.62 (for PODC, OCPODC and MPODC 
complexes) or two triplets appear at $c a$. 7.40-7.72 (for PCPODC and NPDOC complexes) appears.

Thus, on the basis of elemental analysis, electrical conductance measurements and spectral (electronic, infrared and proton magnetic resonance) data, the following structure can tentatively be assigned to the $\left[\mathrm{Zn}(\mathrm{L})_{2}\right]$ complexes (III).<smiles></smiles>

(III)

\section{ANTIFUNGAL ACTIVITY}

The fungicidal activity of the ligands and their corresponding zinc(II) complexes were evaluated against Aspergillus niger, Aspergillus alternate and Helminthosporium oryzae by the agar plate technique at concentrations of 1000,100 and $10 \mathrm{ppm}$ with triplicate determination in each case. The average percentage inhibition after $96 \mathrm{~h}$ was calculated from the expression: inhibition(\%)- $100(\mathrm{C}-\mathrm{T}) / \mathrm{C}$, where $\mathrm{C}$ and $\mathrm{T}$ are the diameters of the fungus colony in control and test plates, respectively. The recorded results (Table III) lead to the following conclusions:

(a) The compounds show significant toxicity at $1000 \mathrm{ppm}$ against all species of fungi and complexes are more active then their corresponding ligands.

(b) The activity decreases on dilution.

(c) The best activity was noted for zinc(II) compound with ligand with $\mathrm{R}=2-\mathrm{CI}$.

(d) The dithiocarbazinates and their corresponding complexes are more active against $A$. niger than $A$. alternata and $H$. oryzae.

Table III

Fungicidal Screening Data

\begin{tabular}{|l|ccccccccc|}
\hline \multirow{2}{*}{ Compound } & \multicolumn{9}{c}{ Fungicidal inhibition (\%) } \\
& \multicolumn{3}{c}{ A. niger } & \multicolumn{9}{c|}{ A. alternate } & \multicolumn{3}{c|}{ H. oryzae } \\
& 1000 & 100 & 10 & 1000 & 100 & 10 & 1000 & 100 & 10 \\
\hline $\mathrm{Zn}(\mathrm{PODC})_{2}$ & 76.2 & 66.8 & 59.0 & 75.2 & 65.8 & 60.0 & 72.1 & 62.4 & 54.8 \\
$\mathrm{Zn}(\mathrm{OCODC})_{2}$ & 92.8 & 80.6 & 70.8 & 85.0 & 72.8 & 68.5 & 82.8 & 68.9 & 60.2 \\
$\mathrm{Zn}(\mathrm{PODC})_{2}$ & 80.6 & 70.2 & 65.6 & 82.1 & 69.4 & 66.8 & 78.6 & 62.8 & 60.1 \\
$\mathrm{Zn}(\mathrm{MODC})_{2}$ & 78.6 & 68.2 & 60.8 & 79.2 & 64.2 & 60.1 & 75.2 & 62.1 & 58.6 \\
$\mathrm{Zn}(\mathrm{NODC})_{2}$ & 75.6 & 60.8 & 56.4 & 74.2 & 58.8 & 54.6 & 74.8 & 60.5 & 56.0 \\
\hline
\end{tabular}




\section{ANTIBACTERIAL ACTIVITY}

The antibacterial activity of the complexes together with parent ligands has been screened against Grampositive Bacillus subtilis and Gram-negative Escherichia coli by the paper disk plate method at $1000 \mathrm{ppm}$ concentration. The inhibition zone ( $\mathrm{mm}$ ) around each disk was measured after $24 \mathrm{~h}$ and the results of these studies are listed in Table IV. It is clear from the screening data that the complexes are slightly more toxic than the parent ligands. The compounds are found to be slightly more toxic against $E$-coli as compared to $B$. subtilis.

Table IV

Bactericidal Screening Data

\begin{tabular}{|c|cc|}
\hline Compound & \multicolumn{2}{|c|}{ Diameter of inhibition zone (mm) } \\
& B. subtilis & E. coli \\
\hline $\mathrm{Zn}(\mathrm{PODC})_{2}$ & 12 & 13 \\
$\mathrm{Zn}(\mathrm{OCODC})_{2}$ & 18 & 20 \\
$\mathrm{Zn}(\mathrm{PODC})_{2}$ & 16 & 18 \\
$\mathrm{Zn}(\mathrm{MODC})_{2}$ & 13 & 15 \\
$\mathrm{Zn}(\mathrm{NODC})_{2}$ & 14 & 15 \\
\hline
\end{tabular}

The synthesized compounds were found to have remarkable bacterial and fungicidal properties; it is however interesting that the biological activity is enhanced upon complexation with the zinc ion. The enhanced activity of metal complexes compared to ligands may be due to the sulphur atoms around the central metal ion arising from chelation. Such an increased activity for the metal chelates as compared to free ligands can be explained on the basis of chelation therapy. Chelation considerably reduces the polarity of metal ion because of the partial sharing of its positive charge with the donor groups and possible p-electron delocalization over the chelate ring. Such chelation could increase the lipophilic character of the central metal atom, which subsequently favours permeation through the lipid layer of cell membrane.

\section{REFERENCES}

1. M.R.L. Oliveira, G.J. Perpetuo, J. Janczak, M.M.M. Rubinger, Polyhedron, 26, 163(2007).

2. D. Coucouvanis, Prog. Inorg. Chem., 11, 233(1970).

3. J. Willemse, J.A. Cras, J.J. Steggerda and C.P. Kijers, Structure and Bonding, 28, 83(1976).

4. M.M. Jones, I.T. Burka, M.E. Hunter, M.A. Basinger, G. Campo and A.D. Weaver, J. Inorg. Nucl. Chem., 42, 775(1980).

5. B. Macias, J.J. Criado, M.V. Vaquero, M.V. Villa and M. Castillo, J. Inorg. Biochem., 42, 17(1991). 
6. O.P. Pandey, S.K. Sengupta and S.C. Tripathi, Rev. Roumaine Chemie, 12, 145(1987).

7. S. Srivastava, V. Srivastava, O.P. Pandey and S.K. Sengupta, Thermochim. Acta, 240, 101(1994).

8. E. Humeres, N.A. Debacher, M.M. de S. Sierra, J. Org. Chem., 64, 1807(1999).

9. A.K. Malik, W. Faubel, Pest. Sci., 55, 965(1999).

10. A. Golcu, Transition Met. Chem., 31, 405(2006).

11. A. Bhatt, S.K. Sengupta and O.P. Pandey, Indian J. Chem., 40A, 994(2001).

12. O.P. Pandey, S.K. Sengupta and S.C. Tripathi, Monatsh. Chemie, 116, 431(1985).

13. K.H. Al-Obaidi, B.F. Ali, R. Abu-El-Halawa and A. Abo-Amer, Transition Met. Chem., 29, 804(2004).

14. P. Gomez-Saiz, J. Garcia-Tojal, M.A. Maestro, J. Mahia, F.J. Arnaiz and T. Rojo, Polyhedron, 21, 2257(2002).

15. H. Singh, L.D.S. Yadav and S.B.S. Mishra, J. Inorg. Nucl. Chem., 43, 1701(1981).

16. S.K. Sengupta, O.P. Pandey, A. Rai and A. Sinha, Spectrochim. Acta, Part A, 65, 139(2006).

17. G.J.M. Vander kerk, M.H. Raalte and A.K. Sijpesteijn, Nature, 176, 308(1955).

18. K.K.M. Yusuff, K.M. Basheer and M. Gopalan, Polyhedron, 2, 839(1983).

19. A.C. Fabretti, F. Forghieri, A. Giusti, C. Preti and G. Tosi, Inorg. Chim. Acta, 86, 127(1984).

20. R.S. Amim, M.R.L. Oliviera, J. Amim Jr., V.M. DeBellis, Transition Met. Chem., 31, 1071(2006).

21. J. Chatt, L.A. Duncanson and L.M. Venanzi, Nature, 177, 1042(1956).

22. K. Nakamoto, J. Fujita, R.A. Condrate and Y. Morimoto, J. Chem. Phys., 39, 423(1963).

23. El. M. Jouad, M. Allain, M.A. Khan, G.M. Bouch, Polyhedron, 24, 327(2005).

24. M.V. de Almeida, W.A. Alves, A.M.D.C. Ferreira, A. Paduan-Filho, Transition Met. Chem., 29, 382(2004). 
